\title{
Process similarity and the simultaneous acquisition retention phenomenon
}

\author{
BENTON J. UNDERWOOD and ARNOLD M. LUND \\ Northwestern University, Evanston, Illinois 60201
}

\begin{abstract}
When two or more lists are learned simultaneously, long-term recall is higher than when lists are learned singly. This phenomenon is called SARP. In the present study, SARP was examined as a function of process similarity. Three lists of 10 items each were learned simultaneously. When these three lists were of the same type (all serial lists, all paired associate lists, or all free recall lists), process similarity was said to be high, whereas when three different list types were learned simultaneously, process similarity was said to be low. Original learning was carried to a criterion of 8 of 10 correct responses on a given trial, with recall taken after $24 \mathrm{~h}$. All three list types produced SARP, but its magnitude was independent of process similarity.
\end{abstract}

If two or more verbal lists are learned simultaneously, the 24-h recall of these lists is better than the recall for a list learned alone (Underwood \& Lund, 1979). This has been identified as the simultaneous acquisition retention phenomenon, or SARP. At the present time, very little is known about the limiting conditions of SARP. The evidence from the original study suggested that the simultaneous acquisition of several lists somehow reduced the interference from extraexperimental sources. To say this another way, simultaneous learning of the lists increased the discrimination between the lists and other potentially interfering habits. If this is true, any independent variable that facilitates the discrimination among tasks learned in the laboratory might also be expected to facilitate the discrimination between the tasks learned in the laboratory and potentially interfering associations previously learned outside the laboratory.

It has been shown that the discrimination among lists learned in the laboratory is influenced by process similarity (Underwood, 1977, Experiment 13). By process similarity, we mean the degree to which the learning processes underlying two or more tasks are similar. If all lists learned are serial lists, or all paired associate lists, or all free recall lists, process similarity is higher than if one list of each type is learned. In the experiment in question (Underwood, 1977), subjects learned either four lists of the same kind (high similarity) or four lists, each of a different kind (low similarity). After acquisition, the subjects were asked to identify the list to which each word belonged. This identification was better with low process similarity than with high process similarity. In the present study, we ask if this discrimination associated with low process similarity will generalize to extraexperimental associations. If it does, the magnitude of SARP should be

This research was supported by Grant MH 32362 from the National Institute of Mental Health. greater when process similarity is low than when it is high.

\section{METHOD}

There were nine conditions in the experiment. Three different types of learning tasks were used, namely, serial lists (S), paired associate lists (P), and free recall lists (F). There were three conditions that were identified as control or single-list conditions. In these conditions, the subject learned a single list to a given criterion, with recall taken after $24 \mathrm{~h}$. These traditional single-list conditions will be identified as Conditions $\mathrm{S}, \mathrm{P}$, and $\mathrm{F}$. In the remaining six conditions, the simultaneous learning of three lists was involved. For three of the conditions, the three lists were different, one being an $S$ list, another a $P$ list, and the third an F list. The three conditions differed only in terms of which list was identified as the critical list: the list that was always recalled first on test trials. Because the lists differed in kind, process similarity is said to be low and the three conditions may be identified as LS-F, where LS stands for low similarity and $F$ stands for the list to be recalled first on test trials (the free recall list). The other two low-similarity conditions were LS-P and LS-S, where $P$ and $S$ represent the critical lists, one being a paired associate list and the other a serial list. It can be seen that a comparison of the recall of the critical list for these three experimental conditions with the recall of the three control lists will determine the magnitude of SARP for each of the three types of lists when process similarity was low.

The final three conditions had high process similarity. In one case, all three lists learned simultaneously were serial lists (Condition HS-S); in another, they were all paired associate lists (Condition HS-P); and in the third case, all were free-recall lists (Condition HS-F). A comparison of the recall of these three critical lists with the recall of the corresponding three control lists defines SARP, and its magnitude may be compared with the magnitude when process similarity was low. If the discriminations set up as a consequence of learning the lists with low process similarity generalize to extraexperimental associations more than is the case when process similarity is high, SARP should be greater with low process similarity than with high process similarity.

\section{Lists}

When lists are learned simultaneously, there must be some way for the subject to discriminate the items in each list from 
those in every other list. In the present experiment, the discriminandum was the number of letters in the words in each list (three, four, or five). Twenty words of each length were chosen such that the Thorndike-Lorge (1944) frequency was equivalent for the three groups. This was done by matching frequencies item by item across the three groups of words. The frequencies covered a broad range. Each of the 20 words began with a different first letter, and all were monosyllabic. The 20 words of each length were paired randomly to form three lists of 10 pairs each. These formed the three $P$ (paired associate) lists. The $S$ (serial) lists were made up of the 10 response terms, as were the $F$ (free recall) lists.

Type of list was confounded with length of words. That is, for Condition S, the three-letter words were always used, and for Conditions LS-S and HS-S the same serial list was the critical list and was always tested first. For Conditions P, LS-P, and HS-P, the lists made up of the four-letter words were used for the control condition (Condition P) and as the critical list for the two experimental conditions (LS-P and HS-P). The five-letter words were used for the control list in free recall learning (Condition F), and the same lists were used as the critical lists in the simultaneous learning for Conditions LS-F and HS-F.

\section{Procedure and Subjects}

The nine conditions were represented by nine groups of 20 subjects each (college students), assigned to conditions from a block-randomized schedule. For the three control conditions, the presentation rate was $1 \mathrm{sec}$. Study trials alternated with test trial under all conditions. The serial lists were tested by having the subject write the words on a prepared data sheet with 10 numbered lines. The subjects were requested to write each word in its appropriate position. The $P$ lists were tested with a sheet on which the 10 stimulus terms were printed with a blank after each. The subjects were asked to fill in the blanks with the appropriate word. The data sheet for the $F$ lists consisted of 10 blank spaces, and the subject was instructed to write as many words as possible in any order. For all conditions, $45 \mathrm{sec}$ were allowed for the test on each list.

The simultaneous learning was handled as in the past (Underwood \& Lund, 1979). The words were presented for study on a memory drum. On any given exposure, one item from each of three lists was shown. A given exposure of the three items was for $6 \mathrm{sec}$. Following the study trial, a test trial was given in which the critical list was always tested first. If the three lists being learned were S lists, the order of the items remained constant from study trial to study trial, but the order of the three words in a given exposure varied randomly. When the three lists were P lists, the order of the words on the study trial varied across three trials, after which the three orders were repeated. The same was true for $\mathrm{F}$ lists. When the three lists had low process similarity, the $S$ lists had the same order from trial to trial and the $\mathrm{P}$ and $\mathrm{F}$ lists had the varied order as described above.

All lists were tested, of course, when simultaneous learning was involved. As noted several times, the critical list (identified by condition name) was always tested first. The two other lists were then tested in succession in exactly the same way as was the critical list. It should be clear that the critical list (the first list tested) is our designation; the subjects were led to believe that one list was as important as the other.

Instructions to the subjects for simultaneous learning included an explanation that the three lists could be distinguished by the number of letters in the words. Furthermore, the test sheets clearly specified the number of letters in the words appropriate for a given test. The differences in the nature of the tests were fully explained when the conditions were LS-F, LS-P, and LS-S. The subjects were instructed to get as many correct responses for each list as possible.

Learning was carried on until the subject produced eight or more correct responses from the critical list on a given trial. The subjects did not know that a criterion had to be reached on a list before learning stopped. They had been instructed that the study and test trials would continue until "I tell you to stop." The subjects returned $24 \mathrm{~h}$ after learning and a test trial was given in the same way as it had been given on the preceding day. Following the test trial, another study-test trial was given to complete the experiment.

In summary, the critical interest is in the recall of three lists learned as single lists, the recall of these same three lists following the simultaneous learning of two additional lists with low process similarity, and the recall of these three lists following simultaneous learning of lists with high process similarity.

\section{RESULTS AND DISCUSSION}

\section{Learning}

The mean numbers of trials required to reach the criterion of eight correct responses are shown in Figure 1 . It will be remembered that the control lists were presented at a $1-\mathrm{sec}$ rate, whereas the rate for the six experimental lists was $6 \mathrm{sec}$. It had seemed from the previous work (Underwood \& Lund, 1979) that these two rates would produce about the same number of trials to reach the criterion. It is obvious from Figure 1 that this was not the case, except for the $\mathrm{P}$ lists. For the $P$ lists, the mean number of trials to learn was about the same for single and simultaneous learning. The $\mathrm{S}$ and $F$ lists required an appreciably greater number of trials

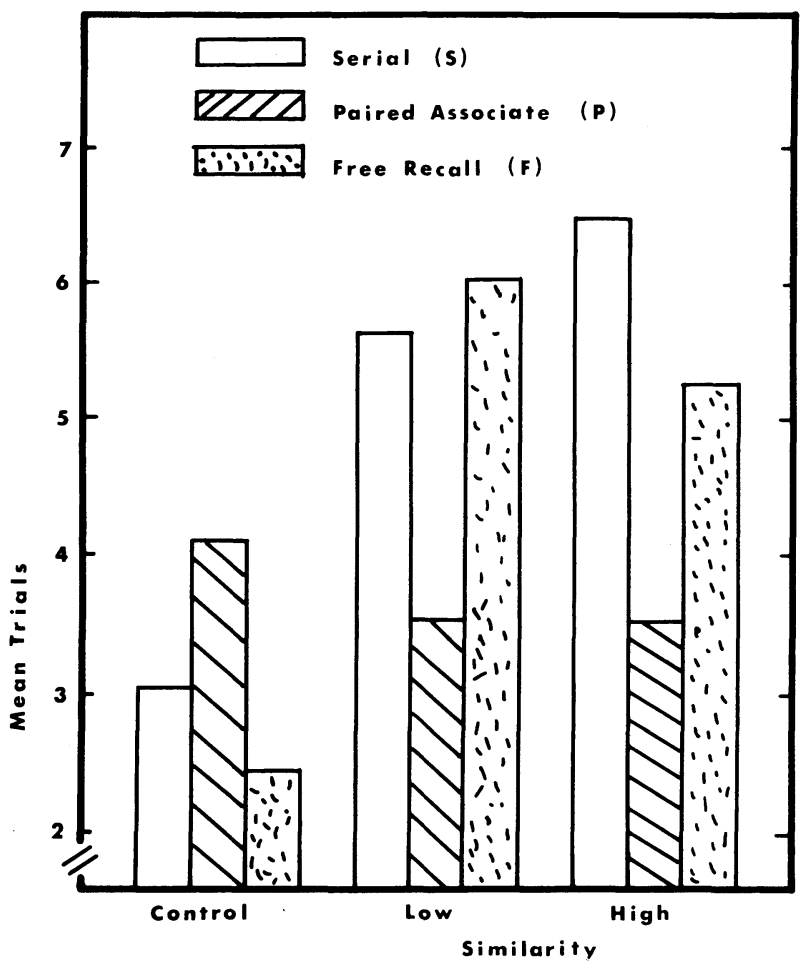

Figure 1. Mean number of trials to reach the criterion of eight correct responses on a given trial as a function of list type (S, P, F) and control vs. experimental lists (low and high similarity). 
to learn when simultaneous learning was involved than when the lists were learned singly.

An analysis of variance was performed on the scores representing the nine conditions. The 5\% significance level was used to reach decisions on the reliability of differences. In the analysis, one variable was the nature of the lists (control, low similarity, high similarity), which we may call similarity, and the other variable was the type of list (S, P, F). Differences given in the figure were statistically confirmed. All calculations involved $\mathrm{MSe}=2.97$ [for similarity, $\mathrm{F}(2,171)=$ 24.10 ; for type of list, $F(2,171)=9.21$; for the interaction, $F(4,171)=11.20$ ]. The interaction reflects the fact that for $\mathrm{S}$ and $\mathrm{F}$ lists the simultaneous learning required more trials to reach criterion than did the control lists, whereas for the $\mathrm{P}$ lists, this was not true. In spite of the differences in rate of learning, the level achieved on the criterion trial was not related to the similarity variable. The only difference was associated with list type. Subjects given $\mathrm{S}$ lists had more correct responses (9.02) on the criterion trial than did the subjects who learned $\mathrm{P}$ lists (8.68) or the $\mathrm{F}$ lists (8.42).

It must be remembered that we were not concerned with differences due to list type. We knew that list type was confounded with number of letters in the words (which might or might not be relevant), but, more important, there are simply no good grounds for making a comparison of learning as a function of list type. We viewed the experiment as consisting of three different and independent tests of SARP as a function of process similarity, the three tests identified as the three different list types. Nevertheless, we were surprised by the differences in learning the control and experimental lists as a function of list type, and we recognized that the differences in trials to reach criterion might be viewed as of consequence in interpreting the retention data. Therefore, we will return to the matter after presenting the recall scores.

\section{Recall}

The mean recall for each of the nine lists after $24 \mathrm{~h}$ is shown in Figure 2. An inspection of the bars for each list type shows that recall in every case was better for the experimental lists (low- and high-similarity lists) than for the control lists. This difference defines SARP. An analysis corresponding to the one performed on the learning data was carried out. Similarity was a reliable variable $[\mathrm{F}(2,171)=6.22, \mathrm{MSe}=3.51]$, as was list type $[\mathrm{F}(2,171)=7.15, \mathrm{MSe}=3.51]$. The latter reflects the fact that recall was higher for the $\mathrm{P}$ lists than for the other two types. The former indicates the better recall of the experimental lists than of the control. The interaction between the two variables produced $F<1$, and this indicates that SARP was independent of process similarity. The above analysis was also carried out using loss scores (correct on the criterion trial minus recall), but the conclusions did not change.

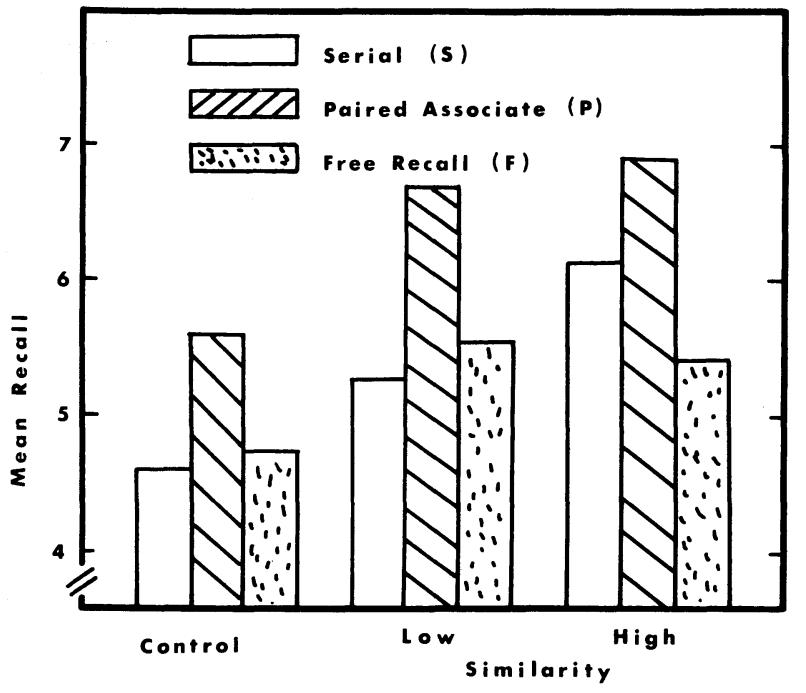

Figure 2. Recall as a function of list type and control vs. experimental lists.

After the recall trial, a further study-test trial was administered. An analysis showed that SARP was not present on this relearning trial, indicating that SARP is a very transitory phenomenon. This was also found in the earlier study (Underwood \& Lund, 1979). The only statistically reliable difference found was that performance was better for the $\mathbf{P}$ lists than for the other two types.

As a final step, we must consider the possibility that SARP might be an artifact for the $S$ and $F$ lists. For these lists, there was an obvious correlation between recall of the critical lists and the number of trials required to learn them. If the results are dependent upon this relationship, the correlations between trials to learn to the criterion and recall should be evident. The four experimental conditions involving serial and free recall were examined. The correlations across subjects between the trials to reach criterion and recall were $-.42, .27$, .11 , and -.11 . None of the correlations was statistically reliable. If, instead of using trials to criterion as the measure of learning, the total number of correct responses given during learning to the criterion is used, the correlations still fail to show a relationship $(.33,-.14,-.38$, .14). We do not believe that SARP, as measured in the $\mathrm{F}$ and $\mathrm{S}$ lists, was due to the fact that the experimental lists required more trials to reach criterion than did the control lists. And, of course, the P lists produced SARP when the number of trials required to reach criterion was essentially equivalent for the experimental and control lists.

The evidence has been interpreted to mean that SARP occurred for all three types of lists but that process similarity did not influence the magnitude of SARP. Low process similarity has been shown to provide better list differentiation than does high process similarity for lists 
learned in the laboratory. In view of the present results, it would now seem that this relationship is not going to be useful in providing an explanation for SARP. If SARP is produced because simultaneous learning produces better differentiation between the lists and conflicting associations learned previously outside the laboratory than does single-list learning, our present results indicate that this differentation is not a function of the process similarity of lists learned simultaneously in the laboratory.

\section{REFERENCES}

Thorndike, E. L., \& Lorge, I. The teacher's word book of 30,000 words. New York: Columbia University Press, 1944. UNDERWOOD, B. J. Temporal codes for memories: Issues and problems. Hillsdale, N.J: Erlbaum, 1977.

UNDERWOOD, B. J., \& Lund, A. M. Retention differences as a function of number of verbal lists learned simultaneously. Journal of Experimental Psychology: Human Learning and Memory, 1979, 5, 151-159.

(Received for publication September 19, 1980.) 